\title{
Dispersion Forces in Methane
}

\author{
By Hendrik N. W. LekKerkerker,* Philippe Coulon and Rene Luyckx
}

Faculteit van de Wetenschappen, Vrije Universiteit Brussel, 1050 Brussels, Belgium

\section{Received 16th February, 1977}

\begin{abstract}
The coefficients of the $R^{-6}$ and $R^{-7}$ terms in the series representation of the dispersion interaction between two methane molecules and between methane and helium, neon and argon are calculated by a variation method.
\end{abstract}

\section{INTRODUCTION}

Buckingham ${ }^{1}$ pointed out that the series representation of the dispersion interaction between molecules, of which at least one does not have a centre of symmetry, contains in addition to the well-known $R^{-6}$ term a $R^{-7}$ term which is due to the mixing of the dipole-dipole and dipole-quadrupole interaction. This $R^{-7}$ term is very interesting in the case of methane interactions since the $R^{-6}$ term is orientationindependent, whereas the $R^{-7}$ term does depend on the orientation of the interacting methane molecules. ${ }^{1}$ Furthermore, the first non-vanishing multipole moment of methane is an octupole moment and the octupole-octupole interaction also varies like $R^{-7}$. To the present stage this octupole-octupole interaction has been considered as the dominant long range orientation dependent interaction in methane. ${ }^{2}$

Although Buckingham ${ }^{1}$ did provide a semi-empirical estimate of the coefficient $C_{7}$ of the $R^{-7}$ term in the dispersion interaction in methane, no calculations of this coefficient from first principles have yet been reported. In view of the fact that in the last few years a number of experimental investigations of the rotational motion of methane in the solid phase ${ }^{3,4}$ as well of methane in rare gas matrices ${ }^{5,6}$ have been reported, we felt it worthwhile to calculate the $R^{-7}$ dispersion interaction between two methane molecules and between methane and helium, neon and argon.

\section{DISPERSION INTERACTIONS AND DYNAMIC POLARIZABILITIES}

The dispersion interaction between two molecules $\mathrm{A}$ and $\mathrm{B}$ is given by those terms in the second order perturbation energy of the electrostatic interaction $V$ between these molecules, in which both $\mathrm{A}$ and $\mathrm{B}$ are excited, i.e.,

$$
U_{\text {disp }}=-\sum_{v^{\prime}, v^{\prime \prime} \neq 0} \frac{\left|\left\langle\psi_{0}^{\mathrm{A}} \psi_{0}^{\mathrm{B}}|V| \psi_{v^{\prime}}^{\mathrm{A}} \psi_{v^{\prime \prime}}^{\mathrm{B}}\right\rangle\right|^{2}}{\left(E_{v^{\prime}}^{\mathrm{A}}-E_{0}^{\mathrm{A}}\right)+\left(E_{v^{\prime \prime}}^{\mathrm{B}}-E_{0}^{\mathrm{B}}\right)} .
$$

Expanding the electrostatic interaction potential $V$ in inverse powers of the intermolecular distance $R$ one obtains

$$
V=V_{\mathrm{dd}}+V_{\mathrm{dq}}+V_{\mathrm{qd}}+\ldots
$$

Here $V_{\mathrm{dd}}$ represents the dipole-dipole interaction which varies like $R^{-3}, V_{\mathrm{dq}}$ represents the dipole (A)-quadrupole (B) interaction which varies like $R^{-4}$ etc. Substituting the series expansion of the interaction potential in (1) one sees that the $R^{-6}$ term in 
the dispersion energy is due to the dipole-dipole interaction, whereas the $R^{-7}$ term arises from the combination of the dipole-dipole and dipole-quadrupole interaction.

Taking into account the symmetry properties of methane it can be shown that the dispersion interaction between a methane molecule A, and a closed shell atom B has the form ${ }^{1}$

$$
U_{\mathrm{disp}}=-\left[\frac{C_{6}}{R^{6}}+\frac{C_{7}}{R^{7}} \cos \theta_{x}^{\mathrm{A}} \cos \theta_{y}^{\mathrm{A}} \cos \theta_{z}^{\mathrm{A}}+\ldots\right] .
$$

Here $\theta_{x}^{\mathrm{A}}$ is the angle between the $x$-axis of the molecular axes system of $\mathrm{A}$ and the vector pointing from the centre of $\mathrm{A}$ to $\mathrm{B}$. The molecular $x, y$ and $z$ axes are perpendicular to the faces of a cube with the $\mathrm{C}$ atom at the centre and the hydrogen atoms at the $(1,1,1),(1,-1,-1),(-1,1,-1)$ and $(-1,-1,1)$ positions. Similarly the interaction between two methane molecules $\mathrm{A}$ and $\mathrm{B}$ can be written as

$$
U_{\text {disp }}=-\left[\frac{C_{6}}{R^{6}}+\frac{C_{7}}{R^{7}}\left\{\cos \theta_{x}^{\mathrm{A}} \cos \theta_{y}^{\mathrm{A}} \cos \theta_{z}^{\mathrm{A}}+\cos \theta_{x}^{\mathrm{B}} \cos \theta_{y}^{\mathrm{B}} \cos \theta_{z}^{\mathrm{B}}\right\}+\ldots\right] .
$$

Starting from eqn (1) and (2) and using the identity

$$
\frac{1}{a+b}=\frac{2}{\pi} \int_{0}^{\infty} \frac{a b}{\left(a^{2}+u^{2}\right)\left(b^{2}+u^{2}\right)} d u
$$

one can express the coefficients $C_{6}$ and $C_{7}$ as integrals of products of dynamic polarizabilities evaluated at imaginary frequencies ${ }^{7}$

$$
\begin{aligned}
& C_{6}=\frac{3}{\pi} \int_{0}^{\infty} \alpha_{x ; x}^{\mathrm{A}}(\mathrm{i} \xi) \alpha_{x ; x}^{\mathrm{B}}(\mathrm{i} \xi) \mathrm{d} \xi \\
& C_{7}=\frac{36}{\pi} \int_{0}^{\infty} \alpha_{x ; y z}^{\mathrm{A}}(\mathrm{i} \xi) \alpha_{x ; x}^{\mathrm{B}}(\mathrm{i} \xi) \mathrm{d} \xi .
\end{aligned}
$$

Here $\alpha_{x ; x}$ is the usual dipole polarizability (which for methane is isotropic) and $\alpha_{x ; y z}$ may be interpreted either as the polarizability for a dipole induced by a field gradient or the polarizability for a quadrupole induced by a uniform field. Thus the calculation of dispersion energies can be reduced to the calculation of the appropriate dynamic polarizabilities. The required dynamic polarizabilities will be calculated by an extension of the Kirkwood variation method. ${ }^{8}$

\section{VARIATIONAL CALCULATION OF DYNAMIC POLARIZABILITIES}

Consider a molecule placed in an external time-dependent field. This leads to the appearance in the hamiltonian of a perturbation operator that can be written as ${ }^{9}$

$$
\begin{aligned}
V(t) & =-\sum_{k=1}^{s} f_{k}(t) A_{k} \\
& =-\frac{1}{2} \sum_{k=1}^{s}\left(f_{0, k} \mathrm{e}^{\mathrm{i} \omega t}+f_{0, k}^{*} \mathrm{e}^{-\mathrm{i} \omega t}\right) A_{k}
\end{aligned}
$$

where $A_{k}$ is an operator that depends on the dynamical variables of the molecule. Owing to the presence of this perturbation the expectation value of the operators $A_{k}$ changes. In first approximation (linear response) this can be expressed in the form

$$
\left\langle A_{k}^{\prime}(t)\right\rangle=\frac{1}{2} \sum_{l=1}^{s}\left[\alpha_{k ; l}(\omega) f_{0, l} \mathrm{e}^{\mathrm{i} \omega t}+\alpha_{k ; l}(-\omega) f_{0, l}^{*} \mathrm{e}^{-\mathrm{i} \omega t}\right] .
$$

* Eqn (6) and (7) are expressed in atomic units, which are used throughout this paper. The atomic unit of length is $0.529 \times 10^{-10} \mathrm{~m}$ and the unit of energy is $4.36 \times 10^{-18} \mathrm{~J}$. 
Here $\langle\ldots\rangle$ indicates an expectation value with respect to the perturbed wave function and the operators $A_{k}^{\prime}$ are defined by

$$
A_{k}^{\prime}=A_{k}-\left\langle A_{k}\right\rangle_{0}
$$

where $\left\langle A_{k}\right\rangle_{0}$ is the expectation value of $A_{k}$ in the unperturbed ground state. The quantities $\alpha_{k ; l}(\omega)$ are called dynamic polarizabilities.

The polarizabilities $\alpha_{k ; l}(\omega)$ can be calculated from the expression ${ }^{7}$

$$
\alpha_{k ; l}(\omega)=\left\langle\psi_{0}\left|A_{k}^{\prime}\right| \chi_{l}^{+}(\omega)+\chi_{l}^{-}(\omega)\right\rangle
$$

where the functions $\chi_{l}^{ \pm}(\omega)$ satisfy the following differential equation

$$
\left(H_{0}-E_{0} \pm \omega\right) \chi_{l}^{ \pm}(\omega)-A_{l}^{\prime} \psi_{0}=0 .
$$

This differential equation is equivalent to the variation principle that requires the functional

$$
L\left[\chi_{l}^{ \pm}(\omega)\right]=\left\langle\chi_{l}^{ \pm}(\omega)\left|H_{0}-E_{0} \pm \omega\right| \chi_{l}^{ \pm}(\omega)\right\rangle-2\left\langle\psi_{0}\left|A_{l}^{\prime}\right| \chi_{l}^{ \pm}(\omega)\right\rangle
$$

to be stationary with respect to variation of $\chi_{l}^{ \pm}(\omega)$. Following Kirkwood ${ }^{8}$ we choose a trial function of the form

$$
\chi_{l}^{ \pm}(\omega)=\sum_{m=1}^{s} c_{m l}^{ \pm}(\omega) A_{m}^{\prime} \psi_{0}
$$

the $c_{m l}^{ \pm}(\omega)$ being variation parameters. Minimizing $L$ [eqn (13)] with respect to these variation parameters one obtains

$$
c_{m l}^{ \pm}(\omega)=\left[\mathbf{N}^{-1} \mathbf{M} \pm \omega \mathbf{I}\right]_{m l}^{-1} .
$$

The matrices $\mathbf{M}$ and $\mathbf{N}$ are defined by

$$
\begin{aligned}
M_{m l} & =\left\langle\psi_{0}\left|A_{m}^{\prime}\left(H_{0}-E_{0}\right) A_{l}^{\prime}\right| \psi_{0}\right\rangle+\left\langle\psi_{0}\left|A_{l}^{\prime}\left(H_{0}-E_{0}\right) A_{m}^{\prime}\right| \psi_{0}\right\rangle \\
N_{m l} & =2\left\langle\psi_{0}\left|A_{m}^{\prime} A_{l}^{\prime}\right| \psi_{0}\right\rangle .
\end{aligned}
$$

It can be shown that the matrix elements $M_{k l}$ can be written as

$$
M_{k l}=\left\langle\psi_{0}\left|\sum_{i}\left(\operatorname{grad}_{i} A_{k}\right)\left(\operatorname{grad}_{i} A_{l}\right)\right| \psi_{0}\right\rangle
$$

(see Appendix 1). The use of this relation materially simplifies the actual calculations. Inserting the variationally determined coefficients $c_{m l}^{ \pm}(\omega)$ in the trial function (14) and substituting this function in (11) one finds for the dynamic polarizability

$$
\alpha_{k ; l}(\omega)=\frac{1}{2}\left[\mathbf{N}\left(\mathbf{c}^{+}(\omega)+\mathbf{c}^{-}(\omega)\right]_{k l} .\right.
$$

Using elementary matrix theory ${ }^{10}$ one can write eqn (15) in a more convenient form for further analysis and calculation

$$
c_{m l}^{ \pm}(\omega)=\sum_{\mu=1}^{s} \frac{R_{m l}^{(\mu)}}{\Delta_{\mu} \pm \omega} .
$$

Here $\Delta_{1}, \Delta_{2}, \ldots, \Delta_{S}$ are the eigenvalues of the matrix $\mathbf{N}^{-1} \mathbf{M}$ and $\mathbf{R}^{(\mu)}$ is the residue matrix corresponding to the eigenvalue $\Delta_{\mu}$. These residue matrices can be expressed in terms of the left and right eigenvectors of $\mathbf{N}^{-1} \mathbf{M}$. The dynamic polarizability can now be written in the form

$$
\alpha_{k ; l}(\omega)=\sum_{\mu=1}^{s} \frac{Z_{k ; l}^{(\mu)}}{\Delta_{\mu}^{2}-\omega^{2}}
$$


where the strength factors $Z_{k l}^{(\mu)}$ are given by

$$
Z_{k ; l}^{\mu}=\Delta_{\mu}\left[\mathbf{N R}^{(\mu)}\right]_{k l} \text {. }
$$

It can be shown that the sum of these strength factors is given by

$$
\sum_{\mu=1}^{s} Z_{k ; l}^{(\mu)}=\left\langle\psi_{0}\left|\sum_{i}\left(\operatorname{grad}_{i} A_{k}\right)\left(\operatorname{grad}_{i} A_{l}\right)\right| \psi_{0}\right\rangle .
$$

Actually this is the exact sum rule for the strength factors of the dynamic polarizability $\alpha_{k ; l}(\omega)$ (see Appendix 2). For the usual dipole polarizability where $A_{k}=$ $A_{l}=\sum_{i=1}^{N} x_{l}$, the sum rule given by eqn (23) reduces to the well known ThomasReiche-Kuhn sum rule which states that the sum (23) equals the number of electrons of the system $N$.

\section{RESULTS AND DISCUSSION}

As discussed in section 2, in order to calculate the dispersion interaction coefficients $C_{6}$ and $C_{7}$ one needs to know the polarizabilities $\alpha_{x ; x}(\omega)$ and $\alpha_{x ; y z}(\omega)$. These can be calculated using the formalism given in the previous section. For the operators $A_{k}$ one has to choose

$$
\begin{aligned}
& A_{1}=\sum_{i=1}^{N} x_{i} \\
& A_{2}=\sum_{i=1}^{N} y_{i} z_{i} .
\end{aligned}
$$

One then obtains

$$
\begin{aligned}
\alpha_{x ; x}(\omega) & =\frac{5.18}{(0.694)^{2}-\omega^{2}}+\frac{4.82}{(0.899)^{2}-\omega^{2}} \\
\alpha_{x ; y z}(\omega) & =\frac{7.72}{(0.694)^{2}-\omega^{2}}-\frac{7.72}{(0.899)^{2}-\omega^{2}} .
\end{aligned}
$$

The matrices $\mathbf{M}$ and $\mathbf{N}$ required in the calculation were evaluated using the S.C.F. wave function for methane given by Snyder and Basch. ${ }^{11}$

From eqn (24) we find for the static dipole polarizability $\alpha_{x ; x}(0)=16.7 a_{0}^{3}$ which is in good agreement with the experimental value ${ }^{12}$ of $17.5 a_{0}^{3}$. For the static dipole-quadrupole polarizability we find from $(25) \alpha_{x ; y z}(0)=" 6.5 a_{0}^{4}$. Buckingham ${ }^{1}$ derived an approximate expression for the static dipole-quadrupole polarizability in terms of bond polarizabilities

$$
\alpha_{x ; y z}(0)=\frac{8}{3 \sqrt{3}}\left(\alpha_{\|}-\alpha_{\perp}\right) R_{0} .
$$

Here $R_{0}$ is the length of the $\mathrm{C}-\mathrm{H}$ bond and $\alpha_{\|}$and $\alpha_{\perp}$ are the polarizabilities parallel and perpendicular to the $\mathrm{C}-\mathrm{H}$ bond. Using the values ${ }^{12} \alpha_{\|}=5.33 a_{0}^{3}, \alpha_{\perp}=3.9 a_{0}^{3}$ and $R_{0}=2.06 a_{0}$ Buckingham's expression yields $\alpha_{x ; y z}(0)=4.5 a_{0}^{3}$ which is of the same order of magnitude as the calculated value.

We now proceed to the calculation of the dispersion interactions between two methane molecules and between methane and helium, neon and argon. In order to do so we need in addition to the dynamic polarizabilities of methane also the dynamic 
polarizabilities of the noble gases. These can of course be calculated with the method given in section 3 . One obtains ${ }^{13}$

$$
\begin{aligned}
& \alpha_{\mathrm{He}}(\omega)=\frac{2}{(1.27)^{2}-\omega^{2}} \\
& \alpha_{\mathrm{Ne}}(\omega)=\frac{10}{(2.47)^{2}-\omega^{2}} \\
& \alpha_{\mathrm{Ar}}(\omega)=\frac{18}{(1.65)^{2}-\omega^{2}} .
\end{aligned}
$$

Using the dynamic polarizabilities (24), (25) and (27) in eqn (6) and (7) one obtains the values for $C_{6}$ and $C_{7}$ that are given in table 1. For the sake of comparison we have included the recommended values for $C_{6}$ given by Dalgarno. ${ }^{14}$

TABLE 1,-DISPERSION INTERACTION COEFFICIENTS FOR METHANE AND SOME NOBLE GASES (IN a.u.)

\begin{tabular}{lcccl}
\multicolumn{1}{c}{$\begin{array}{c}C_{6} \\
\text { interacting } \\
\text { pair }\end{array}$} & $\begin{array}{c}C_{6} \\
\text { present } \\
\text { work }\end{array}$ & $\begin{array}{c}C_{6} \\
\text { recommended } \\
\text { value by } \\
\text { Dalgarno } \\
\text { [ref. (14)] }\end{array}$ & $\begin{array}{c}C_{7} \\
\text { present } \\
\text { work }\end{array}$ & $C_{7} / C_{6}$ \\
$\mathrm{CH}_{4}+\mathrm{CH}_{4}$ & 160 & 150 & 568 & 3.6 \\
$\mathrm{CH}_{4}+\mathrm{He}$ & 15.0 & 14 & 48.8 & 3.3 \\
$\mathrm{CH}_{4}+\mathrm{Ne}$ & 24.1 & 29 & 71.1 & 3.0 \\
$\mathrm{CH}_{4}+\mathrm{Ar}$ & 86.7 & 98 & 272 & 3.1
\end{tabular}

Buckingham not only provided an approximate expression for the static dipolequadrupole polarizability he also provided a semi empirical estimate of the ratio $C_{6} / C_{7}$.

$$
\frac{C_{7}}{C_{6}}=\frac{12 \alpha_{x ; z z}^{\mathrm{CH}_{4}}(0)}{\alpha_{x, x}^{\mathrm{CH}}(0)} .
$$

Using the calculated values for the static polarizabilities one obtains that $C_{7} / C_{6}=4.7$. This is indeed of the same order of magnitude as the calculated values of $C_{7} / C_{6}$ (see table 1).

Let us finally compare the magnitude of the anisotropic $R^{-7}$ dispersion interaction with the octupole-octupole interaction between two methane molecules. The octupole-octupole interaction can be written as ${ }^{2}$

$$
U_{00}=\frac{I_{3}^{2}}{R^{7}} f\left(\omega_{\mathrm{A}}, \vec{\omega}_{\mathrm{B}}\right)
$$

where $I_{3}$ is the octupole moment of the methane molecule and $f\left(\vec{\omega}_{\mathrm{A}}, \vec{\omega}_{\mathrm{B}}\right)$ is a complicated function of the Euler angles (denoted collectively by $\vec{\omega}_{\mathrm{A}}$ and $\vec{\omega}_{\mathrm{B}}$ ) that specify the orientation of the interacting molecules $\mathrm{A}$ and $\mathrm{B}$. The octupole moment can be calculated from the ground state wave function of the methane molecule. Using the S.C.F. wave function given by Snyder and Basch ${ }^{11}$ one finds that $I_{3}=3.94$ a.u. Taking into account that the function $f\left(\vec{\omega}_{\mathrm{A}}, \vec{\omega}_{\mathrm{B}}\right)$ varies roughly between +10 and -10 we see that the anisotropic dispersion interaction and the octupole-octupole interaction in methane are of the same order of magnitude. Thus as has already been suggested by Buckingham the $R^{-7}$ dispersion energy term may contribute significantly to the binding energy of solids containing tetrahedral molecules. The effect of the anisotropic dispersion interaction on the orientational phase transitions in solid methane and the rotational motion of methane in rare gas matrices will be discussed in a future publication. 
The authors are grateful to Dr. F. Mulder for providing them with the necessary elements of the $\mathbf{N}$ matrix.

One of us (R. Luyckx) would like to thank the "Instituut tot aanmoediging van het Wetenschappelijk Onderzoek in Nijverheid en Landbouw (I.W.O.N.L.) " for financial support.

\section{APPENDIX 1}

\section{DEMONSTRATION OF EQN (18)}

One easily verifies that the elements of the matrix $\mathbf{M}$ defined by eqn (16) can be written as

$$
M_{k l}=\frac{1}{2}\left\langle\psi_{0}\left|C_{k+l}-C_{k}-C_{l}\right| \psi_{0}\right\rangle
$$

where the operators $C_{k}, C_{l}$ and $C_{k+l}$ are commutators of the type

$$
C_{k}=\left[A_{k},\left[H_{0}, A_{k}\right]\right] \text {. }
$$

Working out these commutators leads to

Using this result one obtains

$$
C_{k}=\sum_{i=1}^{N}\left(\operatorname{grad}_{i} A_{k}\right)^{2} .
$$

$$
C_{k+l}-C_{k}-C_{l}=2 \sum_{i=1}^{N}\left(\operatorname{grad}_{i} A_{k}\right)\left(\operatorname{grad}_{i} A_{l}\right) .
$$

Substitution of eqn (A1.4) in (A1.1) leads to eqn (18).

\section{APPENDIX 2}

\section{OSCILLATOR STRENGTH SUM RULES}

Using standard time dependent perturbation theory one obtains for the dynamic polarizabilities

$$
\alpha_{k ; l}(\omega)=\sum_{v} \frac{f_{k ; l}^{(v)}}{\left(E_{v}-E_{0}\right)^{2}-\omega^{2}}
$$

where $f_{k ; l}^{(v)}$ is the oscillator strength for the transition to the $v$ th excited state $\psi_{v}$

$$
f_{k: l}^{(v)}=\left(E_{v}-E_{0}\right)\left\{\left\langle\psi_{0}\left|A_{k}\right| \psi_{v}\right\rangle\left\langle\psi_{v}\left|A_{l}\right| \psi_{0}\right\rangle+\left\langle\psi_{0}\left|A_{l}\right| \psi_{v}\right\rangle\left\langle\psi_{v}\left|A_{k}\right| \psi_{0}\right\rangle\right\} .
$$

One easily verifies that

$$
S_{k ; l} \equiv \sum_{v} f_{k ; l}^{(v)}=\frac{1}{2}\left\langle\psi_{0}\left|C_{k+l}-C_{k}-C_{l}\right| \psi_{0}\right\rangle
$$

where the operators $C_{k}, C_{l}$ and $C_{k+l}$ are defined in Appendix 1 [eqn (A1.2)]. Using eqn (A1.4) one obtains

$$
S_{k ; l}=\left\langle\psi_{0}\left|\sum_{i}\left(\operatorname{grad}_{i} A_{k}\right)\left(\operatorname{grad}_{i} A_{l}\right)\right| \psi_{0}\right\rangle .
$$

The sum of the strength factors in the variationally obtained expression for the dynamic polarizability [eqn (21)] yields

$$
\sum_{\mu=1}^{s} Z_{k ; l}^{(\mu)}=\sum_{\mu=1}^{s} \Delta_{\mu}\left[\mathbf{N R}^{(\mu)}\right]_{k l}=M_{k l}
$$

Here we have used the fact that the residue matrices satisfy the identity ${ }^{10}$

$$
\sum_{\mu=1}^{s} \Delta_{\mu} \mathbf{R}^{(\mu)}=\mathbf{N}^{-1} \mathbf{M} \text {. }
$$


Substituting in eqn (A2.5) the expression for $M_{k t}$ given by eqn (18) one obtains

$$
\sum_{\mu=1}^{s} Z_{k ; l}^{(\mu)}=\left\langle\psi_{0}\left|\sum_{i}\left(\operatorname{grad}_{i} A_{k}\right)\left(\operatorname{grad}_{i} A_{l}\right)\right| \psi_{0}\right\rangle .
$$

Thus we see that the variational expression for $\alpha_{k ; l}(\omega)$ satisfies exactly the oscillator strength sum rule.

${ }^{1}$ A. Buckingham, Disc. Faraday Soc., 1965, 40, 232.

${ }^{2}$ H. M. James and T. A. Keenan, J. Chem. Phys., 1959, 31, 12.

${ }^{3}$ W. Press and A. Hüller, Phys. Rev. Letters, 1973, 30, 1207.

${ }^{4}$ W. Press, A. Hüller, H. Stiller, W. Stirling and W. Currat, Phys. Rev, Letters, 1974, 32, 1354.

${ }^{5}$ K. Nishiyama and T. Yamamoto, J. Chem. Phys., 1973, 58, 1001.

${ }^{6}$ K. Kobashi, Y. Kataoka and T. Yamamoto, Canad. J. Chem., 1976, 54, 2154.

${ }^{7}$ C. Mavroyannis and M. J. Stephen, Mol. Phys., 1963, 5, 629.

${ }^{8}$ J. G. Kirkwood, Phys. Z, 1931, 33, 57.

${ }^{9}$ L. Landau and E. Lifshitz, Physique Statistique (Editions Mir, Moscow, 1967), para. 125, 127.

${ }^{10}$ P. Lancaster, Theory of Matrices (Academic Press, New York, 1969), chap. 2.

${ }^{11}$ L. C. Snyder and H. Basch, Molecular Wave Functions and Properties (Wiley, New York, 1972).

12 J. O. Hirschfelder, C. F. Curtiss and R. B. Bird, The Molecular Theory of Gases and Liquids (Wiley, New York, 1954).

${ }^{13} \mathrm{H}$. Lekkerkerker, Ph. Coulon and R. Luyckx, Physica, 1977, in press.

${ }^{14}$ A. Dalgarno, Adv. Chem. Phys., 1968, 12, 143.

(PAPER 7/283) 